\title{
Tone Application Principles and Use Skills in Computer Music Production
}

\author{
Yide Peng \\ School of art, Chengdu University, Chengdu Sichuan, 610106, China
}

Key words: Computer music, Music production, Tone, Application principles, Use skills.

\begin{abstract}
The development history of computer music production technology is still short in China. However, since computer music entered China, it has been widely applied in multiple fields. Nowadays, how to better utilize computer music production technology to optimize leisure and recreation service has become a problem that numerous music producers focus on jointly. Tone is an important factor in computer music production. Only rational selection and application of tone can guarantee the richness, level and quality of computer music production. This paper mainly explores tone application principles and use skills in computer music production, in the hope of offering reference and help for numerous music producers.
\end{abstract}

\section{Introduction}

The forms of tone are abundant, diversified and varied. The sound from different object vibration has different characteristics. Based on the analysis of tone concept in physics, we can know that tone is generated by object vibration. Different vibration frequency will bring entirely different tones so as to make people generate different feelings. In the production process of computer music, rational selection and application of tone can gain good effects, optimize music production quality and promote computer music to have better appreciation property and artistry.

\section{Concept and development of computer music}

The tone can highlight the essential characteristics of the object producing the sound. Different objects producing the sound have different characteristics when making a sound. The texture and structure of objects producing the sound can decide the tone, so people differentiate different musical instruments through distinguishing the tone ${ }^{[1]}$. From Zhou Dynasty, the musical instruments classified according to eight categories of sounds emerged. With the development of social civilization, the quality of tone has received more and more attention. The appearance of computer music and other music forms accords with the demand of era development.

Computer music is produced with the help of computer technology and computer equipment. In a generalized sense, computer music refers to creating, performing and playing music with computer technology and computer equipment. In a narrow sense, computer music refers to controlling digital interface of music equipment or digital audio equipment to produce and play music through programming with the computer. On the whole, computer music production and playing need the support of computer equipment, digital interface of music equipment, digital audio technology, synthesizer, sampling apparatus and so on. Computer music presents the features different from other music in terms of melody, harmonic, musical form structure and orchestration. The production process of computer music involves acoustics, recording, editing, electronics, musical instrument, computer technology and temperament etc. Hence, it is an applied music usbejct. Through analysis of essential features of computer music, we can know that it includes such aspects as composition, 
commanding, performing and recording. The integration of music art and electronic information technology can highlight the artistry and appreciation property of computer music ${ }^{[2]}$. Based on the overview of computer music, it is known that music producers must master computer technology and own solid music foundation in order to produce computer music. In this way, they can proficiently operate acoustic instrument software, control various kinds of electronic equipment, edit and modify the tone, use all kinds of plug-in and perfectly produce music works with multi-voice and multi-tone according to music features and performance rules.

In the production process of computer music, the ultimate purpose of computer music creators is to create music with the help of new technology and new equipment. With the help of computer equipment and technology, music producers can create the music with different styles, such as popular music, country music, Chinese folk music, jazz music, Chinese traditional opera music and symphony ${ }^{[2]}$. The emergence of computer music greatly enriches the expression forms of music, so computer music has great advantages in music production. With multiples forms of tones, computer music can provide more options for music producers so as to let music producers create more wonderful sounds. In accordance with different waveforms, the tones of these sounds can be classified into the tone of orchestral instruments, the tone of national instruments and electronic synthetic tone etc.

In the production process of computer music, digital interface technology of music equipment used by music producers refers to a protocol technique which allows different types of equipment to carry out data exchange. It is also a kind of standard which is designed by computer music equipment manufacturers in order to make keyboards and synthesizers produced by different enterprises cooperative and compatible with each other. In fact, digital interface technology of music equipment is MIDI (Musical Instrument Digital Interface). It connects two or more components in the system so as to allow different types of equipment to carry out data exchange. With the help of MIDI, the equipment with MIDI such as the synthesizer, sound source and computer in the system can implement data exchange. Meanwhile, under the function of the synthesizer, "musical notes" performed by the keyboard can be transmitted to another synthesizer from one synthesizer to achieve the superposition of two different sounds. The superposition of two different sounds can generate another new tone. In this way, music players can liberate their hands and gain the same performance effect, without the need of playing on different synthesizers with both hands. Nowadays, MIDI data have formed specific norms through the development for many years. The data content changes to change control data, exclusive information data and synchronous information data etc. from simple notes.

\section{Main tone application principles in computer music production}

The tone refers to objective attribute of the sound subjectively perceived by the audiences, and it is the "color" of sound. Synthesized tone is different from traditional tone in terms of vocalism principle, and it can influence the steps and process of music production. Next, this paper simply analyzes the main tone application principle in computer music production. The quality and level of computer music production can be ensured so as to get a better effect only when the main principles of tone application in computer music production.

\section{Paying attention to tone balance of computer music}

Different tones have obvious differences in characteristic performance. In the process of voice arrangement, too intensive arrangement will give rise to servile impacts in the performance effect, and it is difficult to gain relatively ideal performance effect. It is known from harmonics analysis that harmonics stresses rational density. It is required to make sure theme voice balances with the accompaniment voice in the aspect of volume, and rationally allocate middle voices. During allocating theme, melody, accompaniment voice, low voice and voices of percussion instruments, strong and weak voices should be rational to get the ideal sound effect ${ }^{[3]}$. 


\section{Paying attention to controlling tone type and scale of computer music}

In the production process of computer music, tone arrangement has certain principles. In tone arrangement process, small and medium scale of orchestra should serve as the reference. Seeing from sound source, the final music played by both software sound source and hardware sound source has rich tone characteristics, but the waveform is still stored in an electronic way and the music is played in the form of audio oscillation in storage and playing aspects. Thus, sound generation ways and features are not same. The contrast ratio and layering feeling of various voices have obvious differences with real orchestra, national band and electroacoustic band, and the former is significantly greater than the latter. Besides, once the number of voices is large and the lines are dense, the final sound effect is barely satisfactory, though sound image arrangement is enhanced. The result of mixing multiple sounds is chaotic, fuzzy and turbid, and the performance effect is very bad. Thus, music producers should pay attention to controlling tone types and scale during applying tone in computer music production so as to avoid the above problems.

\section{Paying attention to avoid misuse of percussion instruments}

In the production process of computer music, although the tone types generated by percussion instruments are rich, music producers still should note the use rationality of percussion instruments when applying the tone to avoid misuse of percussion instruments and the impacts on the final sound effect. In the past orchestral instrument arrangement process, the replacement of rhythm voice with new percussion instruments was still a trend. Thus, certain norms should be abided by to avoid excessive use and the impacts on the final sound effect. Even if most popular music involves modern percussion instruments in the production process, it is still required to pay attention to the use rationality of percussion instruments so as to create the proper style of popular music, enrich the shocking power and infection of popular music and highlight the mood of popular music.

\section{Main skills about tone application in computer music production}

With the development of science and technology, people's sparetime life becomes richer and richer, and their art pursuit and aesthetic demands have become stronger and stronger. Music as a kind of art expression form exists everywhere in people's life. The integration of music and computer technology not just adds color in people's life, but also promotes people to get favorable spiritual enjoyment. Hence, improvement of computer music production quality and level with effective means complies with the demands of era and the public. Music work creators should focus on the quality and effect of computer music production and enhance exploration and utilization of tone use skills.

\section{Skills to use synthetic tone in terms of background}

In music production process, background music is needed to create the atmosphere. Different pad can enrich melody layer, low pitch layer and harmonic layer etc. ${ }^{[4]}$ In the production process of computer music, the use of pad tone can gain the richer sound effect. To be specific, in the process of creating a song, although the use of high frequency and medium frequency can get a good effect, the effect of low frequency is not ideal enough and then New Age tone in Hypersonic is selected. But the practice has proven that the effect of direct use of New Age tone in Hypersonic is not ideal. To get the fade-in feeling, relevant parameters may be adjusted properly, such as enhancing Re-verb time and Wet, removing the head, adjusting Velocity and Volume and mixing it with toll, whistling sound and synthetic orchestral music etc.

\section{Skills to use conventional tone}

In the production process of computer music, music producers should note the melody and the influence of different strength on the tone in tone selection process so as to pay attention to rational combination of strength, create different music in combination of different emotions and make the audiences feel ups and downs of the song and gain different feelings. In addition, music producers 
also should note the influence of music time value on the tone and combine the musical instruments with different tone features to choose corresponding use skills. For example, string instruments have certain advantage in sound extension, so they may be applied in harmonic background layer. The sound extension of nylon guitar is short, and it can generate unexpected effect during discomposing the chord. So, it can serve as harmonic rhythm ${ }^{[5]}$.

\section{Skills to use tone combinations}

In the process of using tone combinations, firstly, the definition of various voices should be noted, and clear layers and sound should be guaranteed. Secondly, music producers need to control, volume balance with the adjustment button. Finally, in the aspect of tone difference complementation, music producers should be good at utilizing characteristics of different musical instruments to achieve tone difference complementation. For instance, the tone of cello needs to be highlighted in the music production process, but there are no more tones of cello that could be chosen. Then, Bason or horn may be used to obtain a good sound effect. Thus, to guarantee correct use of tones, music producers should have a comprehensive understanding of tone nature and sound effect. On this basis, music producers should clearly know the sound effects of different tone combinations.

\section{Skills to non-conventionally use conventional tones}

In the production process of computer music, it is required to note that simulation of natural and real musical instrument tone is not the sole purpose of computer music production. Computer music producers should give full play to the advantages of computer music production and strive to get the better sound effect than real performance. Therefore, during using the tone in the production process of computer music, melody situation expression and emotion should be noted. Conventional tones may be used unconventionally in the specific situation so as to create the corresponding atmosphere. For example, double sound in large group and $1^{\text {st }}$ large group in computer music is low, and can create tense and serious atmosphere, so they are suitable for being used in army marching music production.

\section{Conclusions}

In conclusion, under the background of continuous social development, computer music gets further development. The tones are enriched to certain degree and have broader development space. Thus, music producers should stick to the tone application principles in computer music production process, and apply computer equipment and technology to create high-level music works with open thinking.

\section{References}

[1] Yang Dehua, Zhou Hui, Zhou Caiqiu et al., Study on Tone Application Skills in Computer Music Production, Music Works, 2014(11):146-148.

[2] Lin Feng, Study on Tone Application Skills in Computer Music Production, Northern Music, 2016,36(10):195.

[3] Ye Yao, Comparison of Computer Music Production Ways and Traditional Music Production Ways, Popular Literature, 2015(4):158.

[4] Meng Kun, Study on Application of Modern Technology in Computer Music Production, Music Works, 2015(6):129-130.

[5] Liu Zikai, Overview of Computer Music Production and Soft Sound Source, Popular Literature, 2012(18):19. 